\title{
Constraints on SUSY Seesaw Parameters from Leptogenesis and Lepton Flavor Violation
}

\author{
F. Deppisch ${ }^{1,2 *}$, H. Päs $s^{1,3 \dagger}$, A. Redelbach ${ }^{4 \ddagger}$, R. Rückl ${ }^{1 \S}$ \\ ${ }^{1}$ Institut für Theoretische Physik und Astrophysik \\ Universität Würzburg \\ D-97074 Würzburg, Germany \\ 2 Deutsches Elektronen-Synchrotron DESY \\ D-22603 Hamburg, Germany \\ 3 Department of Physics 85 Astronomy, \\ University of Hawaii at Manoa, 2505 Correa Road, Honolulu, HI 96822, USA \\ ${ }^{4}$ Gesellschaft für Schwerionenforschung \\ Planckstraße 1, D-64291 Darmstadt, Germany
}

\begin{abstract}
We study the constraints on a minimal supersymmetric seesaw model imposed by neutrino data, charged lepton flavor violation, thermal leptogenesis and perturbativity. We show that it is possible to constrain the three heavy Majorana neutrino masses as well as the complex Yukawa coupling matrix. Our results provide a first step towards a seesaw benchmark model for further phenomenological studies and model building.
\end{abstract}

\footnotetext{
*E-mail: deppisch@physik.uni-wuerzburg.de

${ }^{\dagger}$ E-mail: paes@phys.hawaii.edu

${ }^{\ddagger}$ E-mail: A.Redelbach@gsi.de

$\S$ E-mail: rueckl@physik.uni-wuerzburg.de
} 


\section{Introduction}

The discovery of neutrino masses and mixings in neutrino oscillation experiments calls for an extension of the Standard Model (SM) of particle physics. The most elegant way to implement the required neutrino masses into the SM is via the seesaw mechanism [1]. Three right-handed neutrinos are added to the SM particle content, which, being singlets under the SM gauge symmetry, acquire heavy Majorana masses. By integrating out these right-handed Majorana neutrinos, light masses for the left-handed neutrinos are generated naturally. In a supersymmetric version of this mechanism, the lepton flavor violation present in the neutrino sector is transmitted also to the sleptons inducing charged lepton flavor violation (LFV). We thus focus in this work on a supersymmetric seesaw model. In the framework of degenerate right-handed neutrinos, LFV processes in the SUSY seesaw model were studied in [2, 3] (see also [4, 5, 6] for further phenomenological studies on LFV in processes at low energies).

Another particularly attractive feature of the seesaw mechanism is that it offers a natural possibility to generate the observed baryon to photon ratio in the universe [7, 8],

$$
\eta_{B}=\frac{n_{B}-n_{\bar{B}}}{n_{\gamma}}=(6.3 \pm 0.3) \cdot 10^{-10} .
$$

This process is known as leptogenesis [9] and utilizes the fact that Majorana neutrinos do not carry a conserved lepton number. First, a lepton number asymmetry is generated by outof-equilibrium decays of the heavy right-handed Majorana neutrinos and sneutrinos (in the following subsumed as neutrinos) in the early universe. This lepton number asymmetry is later on transformed into a baryon number asymmetry via non-perturbative Standard Model processes induced by finite temperature transitions between topologically distinct vacua separated by the sphaleron barrier. If the right-handed neutrinos are generated by scattering in the thermal bath, leptogenesis provides a particularly straightforward prediction of the baryon asymmetry of the universe. The decay rates of the right-handed neutrinos and washout processes depend on their masses and Yukawa couplings. Hence the observed value of $\eta_{B}$ (11) may imply constraints on the seesaw model [10, 11, 12,

In total, the seesaw mechanism introduces 18 new parameters in addition to the Minimal Supersymmetric Standard Model (MSSM) parameters. A major drawback of the scheme is, that only 9 of these parameters describe the three masses, mixing angles and CP phases of the light neutrinos, which are directly accessible to the experiment. The other nine parameters are related to the masses and mixing of the heavy Majorana sector, which may be related to physics at the GUT scale. The parameters enter the renormalization group 
evolution of the neutrino Yukawa couplings and affect the mixing of the slepton sector, which gives rise to observable LFV processes such as $l_{j} \rightarrow l_{i} \gamma$, with branching ratios that depend on the parameters of the heavy Majorana sector.

On the other hand, these parameters enter the prediction of the baryon asymmetry via leptogenesis and thus also affect the lower bound on the reheating temperature of the universe after inflation, which governs the gravitino abundance. Since the decay of longliving gravitinos can dissociate light elements and thus jeopardize the successful predictions of Big-Bang nucleosynthesis, a low reheating temperature is desirable.

In this work we study the constraints on the parameters of the SUSY seesaw model in a mSUGRA framework imposed by the combined neutrino data, successful leptogenesis with low reheating temperature and charged lepton flavor violation. Furthermore, we require that the Yukawa couplings do not become too large so that the perturbative theory holds, and assume hierarchical spectra for both the left- and right-handed neutrinos.

This work is organized as follows. The theoretical framework of the SUSY seesaw model is outlined in Section 2. Section 3 introduces the neutrino data used in our calculations. Leptogenesis is explained in Section 4, followed by a discussion of the gravitino problem in Section 5. Constraints from leptogenesis and charged lepton flavor violation on the seesaw parameters are derived in Section 6 and 7.

\section{SUSY seesaw model}

The simplest supersymmetric version of the seesaw mechanism is described by the superpotential [5]

$$
\mathcal{W}=\mathcal{W}_{M S S M}+\frac{1}{2} \nu_{R}^{c T} M \nu_{R}^{c}+\nu_{R}^{c T} Y_{\nu} l \cdot h_{2}
$$

where $\mathcal{W}_{M S S M}$ describes the MSSM, $\nu_{R}$ denotes the right-handed neutrino singlets and $l$ the

left-handed lepton doublets, $h_{2}$ the Higgs doublet with hypercharge $+\frac{1}{2}$, and $Y_{\nu}$ the matrix of neutrino Yukawa couplings. Below the mass scale of the lightest right-handed Majorana neutrino, the effective superpotential [6] is obtained by integrating out the heavy neutrino fields,

$$
\mathcal{W}_{e f f}=\mathcal{W}_{M S S M}+\frac{1}{2}\left(Y_{\nu} l \cdot h_{2}\right)^{T} M^{-1}\left(Y_{\nu} l \cdot h_{2}\right)
$$

After electroweak symmetry breaking, $\mathcal{W}_{\text {eff }}(3)$ leads to the following mass term for the light neutrinos,

$$
m_{\nu}=m_{D}^{T} M^{-1} m_{D}=Y_{\nu}^{T} M^{-1} Y_{\nu}\left\langle h_{2}^{0}\right\rangle^{2} \equiv \kappa\left\langle h_{2}^{0}\right\rangle^{2}
$$


where $\left\langle h_{2}^{0}\right\rangle^{2}=v_{2}^{2} \equiv v^{2} \sin ^{2} \beta$ is the vacuum expectation value (v.e.v.) of the relevant Higgs field and $m_{D}=Y_{\nu} v_{2}$ is the neutrino Dirac mass matrix. Here it has been assumed that the scale of $m_{D}$ is much smaller than the scale of the heavy neutrino mass matrix, $m_{D} \ll M$. This corresponds to the so called type I seesaw mechanism, where any direct (type II) contributions to the light neutrino mass matrix are neglected. Such terms can be generated, e.g. from v.e.v's of SU(2)-triplet Higgs fields.

It is convenient to work in the flavor basis in which the charged lepton Yukawa matrix is diagonal, so that the symmetric matrix $\kappa$ is diagonalized by the unitary Maki-NakagawaSakata (MNS) matrix $U$,

$$
U^{T} \kappa U=\operatorname{Diag}\left(\kappa_{1}, \kappa_{2}, \kappa_{3}\right) \equiv D_{\kappa},
$$

the light neutrino mass eigenstates being given by

$$
m_{i}=v_{2}^{2} \kappa_{i}, \quad i=1,2,3 .
$$

If one chooses $\kappa_{i} \geq 0, U$ can be written as

$$
U=V \cdot \operatorname{Diag}\left(e^{-i \phi / 2}, e^{-i \phi^{\prime} / 2}, 1\right),
$$

where $\phi, \phi^{\prime}$ are Majorana phases and $V$ can be parametrized in the standard CabibboKobayashi-Maskawa form,

$$
V=\left(\begin{array}{ccc}
c_{13} c_{12} & c_{13} s_{12} & s_{13} e^{-i \delta} \\
-c_{23} s_{12}-s_{23} s_{13} c_{12} e^{i \delta} & c_{23} c_{12}-s_{23} s_{13} s_{12} e^{i \delta} & s_{23} c_{13} \\
s_{23} s_{12}-c_{23} s_{13} c_{12} e^{i \delta} & -s_{23} c_{12}-c_{23} s_{13} s_{12} e^{i \delta} & c_{23} c_{13}
\end{array}\right)
$$

with $c_{i j}=\cos \theta_{i j}$ and $s_{i j}=\sin \theta_{i j}$.

From the relations (44) and (5), and working in the basis where $M$ is diagonal, $M=$ $\operatorname{Diag}\left(M_{1}, M_{2}, M_{3}\right)=D_{M}$, one obtains

$$
U^{*} D_{\kappa} U^{\dagger}=\left(R^{T} \sqrt{D_{M}^{-1}} Y_{\nu}\right)^{T}\left(R^{T} \sqrt{D_{M}^{-1}} Y_{\nu}\right)
$$

where $R$ is an arbitrary complex orthogonal matrix [6], $R^{T} R=R R^{T}=1$. The neutrino Yukawa coupling matrix can thus be expressed as

$$
Y_{\nu}=\sqrt{D_{M}} R \sqrt{D_{\kappa}} U^{\dagger} .
$$

The complex orthogonal matrix $R$ can be parametrized by three complex angles $\theta_{i}=x_{i}+i y_{i}$ :

$$
R=\left(\begin{array}{ccc}
\hat{c}_{2} \hat{c}_{3} & -\hat{c}_{1} \hat{s}_{3}-\hat{s}_{1} \hat{s}_{2} \hat{c}_{3} & \hat{s}_{1} \hat{s}_{3}-\hat{c}_{1} \hat{s}_{2} \hat{c}_{3} \\
\hat{c}_{2} \hat{s}_{3} & \hat{c}_{1} \hat{c}_{3}-\hat{s}_{1} \hat{s}_{2} \hat{s}_{3} & -\hat{s}_{1} \hat{c}_{3}-\hat{c}_{1} \hat{s}_{2} \hat{s}_{3} \\
\hat{s}_{2} & \hat{s}_{1} \hat{c}_{2} & \hat{c}_{1} \hat{c}_{2}
\end{array}\right)
$$


with $\hat{c}_{i} \equiv \cos \left(\theta_{i}\right)$ and $\hat{s}_{i} \equiv \sin \left(\theta_{i}\right)$.

We focus on the simplest scenario, where the right-handed neutrinos are hierarchical ${ }^{1}$,

$$
M_{1} \ll M_{2} \ll M_{3},
$$

and thus the baryon asymmetry is generated essentially in the decays of the lightest righthanded neutrino with mass $M_{1}$. Consequently, we also assume a hierarchical left-handed neutrino spectrum,

$$
m_{1} \ll m_{2} \ll m_{3},
$$

since Yukawa couplings and hierarchical Majorana masses would have to conspire in an unnatural way to lead to a degenerate spectrum for the light neutrinos.

To zero order in the small ratios $M_{1} / M_{3}, M_{2} / M_{3}$ and $m_{1} / m_{3}$ the Yukawa coupling (10) is given by

$$
Y_{\nu} \simeq \frac{\sqrt{M_{3} m_{3}}}{v \sin \beta} \operatorname{Diag}(0,0,1) \cdot R \cdot \operatorname{Diag}\left(0, \sqrt{\frac{m_{2}}{m_{3}}} e^{i \phi^{\prime} / 2}, 1\right) V^{\dagger},
$$

with $m_{2} \simeq \sqrt{\Delta m_{12}^{2}}$ and $m_{3} \simeq \sqrt{\Delta m_{23}^{2}}$, defined through the mass squared differences measured in neutrino oscillation experiments. Inserting the parametrization (11) for $R$ one finally arrives at

$$
\left(Y_{\nu}\right)_{i j} \simeq \frac{\sqrt{M_{3} m_{3}}}{v \sin \beta} \hat{c}_{2} \delta_{i 3}\left(\hat{s}_{1} \sqrt{\frac{m_{2}}{m_{3}}} e^{i \phi^{\prime} / 2} V_{j 2}^{*}+\hat{c}_{1} V_{j 3}^{*}\right)
$$

Before proceeding, we briefly summarize the renormalization group evolution of neutrino Yukawa couplings from $m_{Z}$ to the GUT-scale $M_{G U T}$ for non-degenerate seesaw scales [15]. Below $M_{1}$, the heavy Majorana neutrinos are decoupled, so that $Y_{\nu}=0$ and only the effective light neutrino mass matrix $\kappa$ evolves, starting from the input value $\kappa\left(m_{Z}\right)=U^{*} D_{\kappa} U^{\dagger}$. At the $M_{1}$-threshold, the corresponding right-handed Majorana neutrino becomes active and one has

$$
\left.\left(Y_{\nu}\right)_{i j}\right|_{M_{1}+}=\left.\delta_{i 1}\left(\sqrt{D_{M}} R \sqrt{D_{\kappa}} U^{\dagger}\right)_{i j}\right|_{M_{1}-},
$$

where $M_{1}+\left(M_{1}-\right)$ denotes the right (left) limit approaching the scale $M_{1}$. Furthermore, using (44), the tree-level matching condition for $\kappa$ at $M_{1}$ is given by

$$
\left.(\kappa)_{i j}\right|_{M_{1}+}=\left.(\kappa)_{i j}\right|_{M_{1-}}-\left.\left(Y_{\nu}^{T}\right)_{i 1} \frac{1}{M_{1}}\left(Y_{\nu}\right)_{1 j}\right|_{M_{1}-} .
$$

Note that the masses of heavy neutrinos also run above the respective mass thresholds.

\footnotetext{
${ }^{1}$ For a discussion of leptogenesis in scenarios with degenerate neutrinos see [13, 14].
} 
Now we continue with the evolution from $M_{1}$ to $M_{2}$ using the input values $\left.\kappa\right|_{M_{1}+},\left.Y_{\nu}\right|_{M_{1}+}$ and $\left.M_{1}\right|_{M_{1}+}$. The matching

$$
\begin{aligned}
\left.\left(Y_{\nu}\right)_{i j}\right|_{M_{2}+} & =\left.\left(Y_{\nu}\right)_{i j}\right|_{M_{2}-}+\left.\delta_{i 2}\left(\sqrt{D_{M}} R \sqrt{D_{\kappa}} U^{\dagger}\right)_{i j}\right|_{M_{2}-}, \\
\left.(\kappa)_{i j}\right|_{M_{2}+} & =\left.(\kappa)_{i j}\right|_{M_{2}-}-\left.\left(Y_{\nu}^{T}\right)_{i 2} \frac{1}{M_{2}}\left(Y_{\nu}\right)_{2 j}\right|_{M_{2}-}
\end{aligned}
$$

at $M_{2}$ is analogous to the matching at $M_{1}$. Above the $M_{2}$ threshold, the $2 \times 2$ submatrix of $M$ receives small off-diagonal elements, so that at the $M_{3}$ scale $M$ has to be diagonalized by an unitary matrix $U_{M},\left.U_{M}^{T} M\right|_{M_{3}-} U_{M}=D_{M}$. The re-diagonalization of $M$ leads to the redefinition $Y_{\nu} \rightarrow U_{M}^{*} Y_{\nu}$. As has been noted in [15], the renormalization group equations (RGE's) are invariant under the transformations that diagonalize $M$. Therefore the matching conditions at $M_{3}$ are given by

$$
\begin{aligned}
\left.\left(Y_{\nu}\right)_{i j}\right|_{M_{3}+} & =\left.\left(U_{M}^{*} Y_{\nu}\right)_{i j}\right|_{M_{3}-}+\left.\delta_{i 3}\left(\sqrt{D_{M}} R \sqrt{D_{\kappa}} U^{\dagger}\right)_{i j}\right|_{M_{3}-}, \\
\left.(\kappa)_{i j}\right|_{M_{3}+} & =0 .
\end{aligned}
$$

Finally, from (20) and (21), we continue the evolution up to the unification scale $M_{X}$.

With $M$ and $Y_{\nu}$ now calculated at $M_{X}$, we now turn to the slepton sector, assuming the mSUGRA universality conditions

$$
m_{\tilde{l}_{L}}^{2}=m_{0}^{2} 11, \quad m_{\tilde{l}_{R}}^{2}=m_{0}^{2} 11, \quad A_{e}=A_{0} Y_{l}, \quad A_{\nu}=A_{0} Y_{\nu},
$$

at $M_{X}$, where $m_{0}$ is the common scalar mass and $A_{0}$ the common trilinear coupling. At lower scales of order of the SUSY threshold, the mass squared matrix of the charged sleptons has the form

$$
m_{\tilde{l}}^{2}=\left(\begin{array}{cc}
m_{\tilde{l}_{L}}^{2} & m_{\tilde{l}_{L R}}^{2 \dagger} \\
m_{\tilde{l}_{L R}}^{2} & m_{\tilde{l}_{R}}^{2}
\end{array}\right),
$$

where $m_{\tilde{l}_{L}}^{2}, m_{\tilde{l}_{R}}^{2}$ and $m_{\tilde{l}_{L R}}^{2}$ are $3 \times 3$ matrices in flavor space, $m_{\tilde{l}_{L}}^{2}$ and $m_{\tilde{l}_{R}}^{2}$ being hermitian. The respective matrix elements are given by

$$
\begin{aligned}
\left(m_{\tilde{l}_{L}}^{2}\right)_{i j} & =\left(m_{L}^{2}\right)_{i j}+\delta_{i j}\left(m_{l_{i}}^{2}+m_{Z}^{2} \cos 2 \beta\left(-\frac{1}{2}+\sin ^{2} \theta_{W}\right)\right), \\
\left(m_{\tilde{l}_{R}}^{2}\right)_{i j} & =\left(m_{R}^{2}\right)_{i j}+\delta_{i j}\left(m_{l_{i}}^{2}-m_{Z}^{2} \cos 2 \beta \sin ^{2} \theta_{W}\right), \\
\left(m_{\tilde{l}_{L R}}^{2}\right)_{i j} & =\left(A_{e}\right)_{i j} v \cos \beta-\delta_{i j} m_{l_{i}} \mu \tan \beta,
\end{aligned}
$$

$\theta_{W}$ being the weak-mixing angle, and $\mu$ the SUSY Higgs-mixing parameter. The first terms on the r.h.s. of (24) - (26) receive the following contributions:

$$
m_{L}^{2}=m_{0}^{2} 1 l+\left(\delta m_{L}^{2}\right)_{\mathrm{MSSM}}+\delta m_{L}^{2}
$$




$$
\begin{aligned}
m_{R}^{2} & =m_{0}^{2} 1 \mathrm{l}+\left(\delta m_{R}^{2}\right)_{\mathrm{MSSM}}+\delta m_{R}^{2}, \\
A_{e} & =A_{0} Y_{l}+\left(\delta A_{e}\right)_{\mathrm{MSSM}}+\delta A_{e} .
\end{aligned}
$$

Here, $\left(\delta m_{L, R}^{2}\right)_{\text {MSSM }}$ and $\left(\delta A_{e}\right)_{\text {MSSM }}$ denote the usual MSSM renormalization group corrections [16, 17] which are flavor-diagonal. In addition, the right-handed neutrinos radiatively induce the flavor non-diagonal terms $\delta m_{L, R}$ and $\delta A_{e}$, resulting from the RGE's

$$
\begin{aligned}
16 \pi^{2} \frac{d \delta m_{L}^{2}}{d \ln \mu} & =m_{L}^{2} Y_{\nu}^{\dagger} Y_{\nu}+Y_{\nu}^{\dagger} Y_{\nu} m_{L}^{2}+2\left(Y_{\nu}^{\dagger} m_{\tilde{\nu}}^{2} Y_{\nu}+m_{h_{2}}^{2} Y_{\nu}^{\dagger} Y_{\nu}+A_{\nu}^{\dagger} A_{\nu}\right), \\
16 \pi^{2} \frac{d \delta m_{R}^{2}}{d \ln \mu} & =0 \\
16 \pi^{2} \frac{d \delta A_{e}}{d \ln \mu} & =2 Y_{e} Y_{\nu}^{\dagger} A_{\nu}+A_{e} Y_{\nu}^{\dagger} Y_{\nu} .
\end{aligned}
$$

For qualitative discussions, we mention here the results in the leading logarithmic approximation,

$$
\begin{aligned}
\delta m_{L}^{2} & =-\frac{1}{8 \pi^{2}}\left(3 m_{0}^{2}+A_{0}^{2}\right)\left(Y_{\nu}^{\dagger} L Y_{\nu}\right), \\
\delta m_{R}^{2} & =0 \\
\delta A_{e} & =-\frac{3 A_{0}}{16 \pi^{2}}\left(Y_{l} Y_{\nu}^{\dagger} L Y_{\nu}\right),
\end{aligned}
$$

with

$$
L_{i j}=\ln \left(\frac{M_{X}}{M_{i}}\right) \delta_{i j} .
$$

In the following we adopt the particle spectrum of the mSUGRA benchmark scenario SPS1a [18] as an illustrative example. This allows us to easily put our work in context with other SUSY analyses such as [19]. While the numerical results depend on this choice, the generic features also apply to other SUSY scenarios with universality conditions such as in (22).

\section{$3 \quad$ Neutrino masses and mixing}

An important requirement on the seesaw model is that the observed light neutrino masses and mixings are reproduced. This is automatically guaranteed in the $R$ matrix parametrization sketched in Section 2 where the light neutrino parameters can be used as input at the electroweak scale in the renormalization group evolution of the Yukawa coupling matrix. In the following, we use the global best fit values of the neutrino oscillation parameters obtained in a three neutrino framework [20] (for a more recent analysis, see [21]) comprising KamLAND, CHOOZ, MACRO, Super-Kamiokande and SNO data as well as the first 
spectral data from the K2K long baseline accelerator experiment. The resulting best fit values are summarized in Tab. 1. In order to indicate the uncertainties from the experimental errors one will have to live with, we have used $2 \sigma$-errors expected from future measurements as explained in [2].

\begin{tabular}{|c|c|c|}
\hline Parameter & best fit & future range \\
\hline \hline $\sin ^{2} \theta_{23}$ & 0.52 & ${ }_{-0.1}^{+0.1}$ \\
\hline $\sin ^{2} \theta_{13}$ & 0.005 & ${ }_{-0.005}^{+0.001}$ \\
\hline $\sin ^{2} \theta_{12}$ & 0.30 & ${ }_{-0.05}^{+0.05}$ \\
\hline$\Delta m_{12}^{2} / 10^{-5} \mathrm{eV}^{2}$ & 6.9 & ${ }_{-0.36}^{+0.36}$ \\
\hline$\Delta m_{23}^{2} / 10^{-3} \mathrm{eV}^{2}$ & 2.3 & ${ }_{-0.9}^{+0.7}$ \\
\hline
\end{tabular}

Table 1: Best-fit values [20] and 2 $\sigma$ C.L. uncertainties expected from future measurements of neutrino oscillation parameters.

For the Dirac phase $\delta$ and the two Majorana phases $\phi$ and $\phi^{\prime}$ introduced in (7), no experimental limits exist (compare, however [22]).

Upper bounds on the absolute mass scale of neutrinos can be obtained from tritium beta decay experiments, neutrinoless double beta decay searches and the neutrino hot dark matter effect on the cosmological large scale structure and the cosmic microwave background [23. Furthermore, generation of the baryon asymmetry (11) via thermal leptogenesis from the decays of the lightest Majorana neutrino $N_{1}$ yields a constraint ${ }^{2}$ on the mass scale of the lightest neutrino [24, 25]:

$$
m_{1}<0.15 \mathrm{eV}
$$

Moreover, as has been mentioned above, a hierarchical right-handed neutrino spectrum strongly favors a hierarchical left-handed neutrino spectrum. We therefore assume for consistency that next-generation double beta decay experiments will not observe neutrinoless double beta decay and restrict our discussion to left-handed neutrinos being lighter than the anticipated double beta decay sensitivity, $m_{1} \lesssim 0.03 \mathrm{eV}$.

\section{Leptogenesis}

Thermal leptogenesis as a mechanism for the generation of the baryon asymmetry of the universe has been discussed extensively in 26, 24, For the sake of completeness we review in

\footnotetext{
${ }^{2} \mathrm{An}$ at least mild hierarchy of right-handed neutrinos is assumed and strong phase cancellations, which may relax this bound, are neglected [14].
} 
the following the most important relations and facts. The baryon to photon ratio generated from out-of-equilibrium decays of the heavy right-handed neutrinos is given by

$$
\eta_{B} \approx d a_{\mathrm{Sph}} \epsilon_{1} \kappa_{f} .
$$

The $C P$ asymmetry $\epsilon_{1}$ generated in the decays of the lightest right-handed neutrino $N_{1}$ is defined as [27]

$$
\epsilon_{1}=\frac{\Gamma\left(N_{1} \rightarrow h_{2}+l\right)-\Gamma\left(N_{1} \rightarrow \bar{h}_{2}+\bar{l}\right)}{\Gamma\left(N_{1} \rightarrow h_{2}+l\right)+\Gamma\left(N_{1} \rightarrow \bar{h}_{2}+\bar{l}\right)} .
$$

In the SUSY seesaw model one also has to consider the supersymmetric versions of these interactions involving e.g. heavy right-handed sneutrinos [27. The $C P$ violation in the decays of the $N_{i}$ arises from the interference of tree-level decay diagrams with vertex and self-energy corrections [28]

$$
\begin{aligned}
\epsilon_{1} & \simeq-\frac{1}{8 \pi} \frac{1}{\left(Y_{\nu} Y_{\nu}^{\dagger}\right)_{11}} \sum_{j \neq 1} \Im m\left(\left(Y_{\nu} Y_{\nu}^{\dagger}\right)_{1 j}\left(Y_{\nu} Y_{\nu}^{\dagger}\right)_{1 j}\right) f\left(\frac{M_{j}^{2}}{M_{1}^{2}}\right), \\
f(x) & =\sqrt{x}\left(\frac{2}{x-1}+\ln \frac{1+x}{x}\right) .
\end{aligned}
$$

For hierarchical heavy Majorana neutrinos $\frac{M_{j}^{2}}{M_{1}^{2}} \gg 1, j \neq 1$, and apart from strong phase cancellations [14, one obtains $f\left(\frac{M_{j}^{2}}{M_{1}^{2}}\right) \simeq 3 \frac{M_{1}}{M_{j}}$, leading to [29, 10]

$$
\epsilon_{1} \simeq-\frac{3}{8 \pi} \frac{1}{\left(Y_{\nu} Y_{\nu}^{\dagger}\right)_{11}} \sum_{j \neq 1} \Im m\left(\left(Y_{\nu} Y_{\nu}^{\dagger}\right)_{1 j}\left(Y_{\nu} Y_{\nu}^{\dagger}\right)_{1 j}\right) \frac{M_{1}}{M_{j}}
$$

As the MNS matrix $U$ drops out in (42), it is clear that non-zero imaginary parts of the $R$ matrix elements are necessary to generate a $C P$ asymmetry.

Substituting the expression (10) into (42) one gets [10]

$$
\epsilon_{1} \simeq-\frac{3}{8 \pi} \frac{M_{1}}{v_{2}^{2}} \frac{\sum_{i} m_{i}^{2} \Im m\left(R_{1 i}^{2}\right)}{\sum_{i} m_{i}\left|R_{1 i}\right|^{2}}
$$

Using furthermore the orthogonality condition $\sum_{i} R_{1 i}^{2}=1$, one approximately has [10, 30]

$$
\left|\epsilon_{1}\right| \lesssim \frac{3}{8 \pi} \frac{M_{1}}{v_{2}^{2}}\left(m_{3}-m_{1}\right) \text {. }
$$

For a hierarchical spectrum of light neutrinos, the $C P$ asymmetry $\epsilon_{1}$ is maximal. On the other hand the relation (44) also implies a lower bound on the $M_{1}$-scale, e.g. if $\epsilon_{1}>10^{-6}$, then $M_{1}>4 \cdot 10^{9} \mathrm{GeV}[10]$. 
The efficiency factor $\kappa_{f}$ takes into account the effects of the washout rate on an initial $(B-L)$ asymmetry by inverse decays, and by $\Delta L=1$ and $\Delta L=2$ scattering processes. A reliable numerical fit for $\kappa_{f}$ for hierarchical light neutrinos in the strong washout regime is given by [26, 24, 31, 32]

$$
\kappa_{f}\left(\tilde{m}_{1}\right) \simeq(1.5 \pm 0.7) \cdot 10^{-2}\left(\frac{10^{-2} \mathrm{eV}}{\tilde{m}_{1}}\right)^{1.1 \pm 0.1}
$$

The generated $(B-L)$ asymmetry strongly depends on the effective neutrino mass

$$
\tilde{m}_{1}=v^{2} \sin ^{2} \beta \frac{\left(Y_{\nu} Y_{\nu}^{\dagger}\right)_{11}}{M_{1}}
$$

For values of $\sqrt{\Delta m_{12}^{2}}<\tilde{m}_{1}<\sqrt{\Delta m_{23}^{2}}$ in the strong washout regime, the predictions of the final baryon asymmetry become independent of initial conditions and have minimal theoretical uncertainties [25, 24, 33].

Finally one also has to take into account the dilution of the asymmetry due to standard photon production from the onset of leptogenesis until the recombination time of photons [25], described by the dilution factor $d=\left(3 g_{\gamma}^{r e c}\right) /\left(4 g_{\gamma}^{M S S M}\right) \simeq 1 / 78$ in the MSSM, where $g_{\gamma}^{M S S M}$ and $g_{\gamma}^{r e c}$ denote the degrees of freedom in the MSSM and at the time of recombination, respectively.

The initial $B-L$ asymmetry is subsequently converted to a baryon asymmetry by sphaleron processes. In the case of the MSSM with three fermion generations and two Higgs doublets and taking into account the chemical potentials of all particle species in the hightemperature phase, one obtains the conversion factor

$$
a_{\mathrm{Sph}}=\frac{8}{23} .
$$

Note that this factor of roughly one third also arises in the SM with one Higgs doublet. Since $\eta_{B}$ is known quite accurately and the effective neutrino mass is given by $\tilde{m}_{1}=$ $\sum_{i} m_{i}\left|R_{1 i}\right|^{2}$, there is a direct relation between $M_{1}$ and the light neutrino masses and $R$ matrix elements,

$$
M_{1} \simeq-\frac{8 \pi}{3} \frac{\eta_{B}}{d a_{\mathrm{Sph}}} \frac{\sum_{i} m_{i}\left|R_{1 i}\right|^{2}}{\sum_{i} m_{i}^{2} \Im m\left(R_{1 i}^{2}\right)} \frac{v_{2}^{2}}{\kappa_{f}} .
$$

Note that (48) is a direct consequence of a successful thermal leptogenesis for hierarchical $M_{i}$ 


\section{Gravitino production and reheating temperature}

A major obstacle of thermal leptogenesis can be the generation of an overabundance of gravitinos which causes serious cosmological difficulties [34]. Since the couplings of the gravitino to ordinary matter are strongly suppressed by the gravitational scale, it has a very long lifetime. Nevertheless, if it is heavier than the Lightest Supersymmetric Particle (LSP), it can decay e.g. radiatively into a photon and a photino. These decays will occur after the big-bang nucleosynthesis $(\mathrm{BBN})$, unless the gravitino is heavier than $\sim 10 \mathrm{TeV}[35$. Among the gravitino decay products are energetic photons which induce electromagnetic cascade processes, thereby spoiling successful BBN. Since the number of gravitinos produced during the reheating epoch is approximately proportional to the reheating temperature $T_{R}$, one can obtain upper bounds on $T_{R}$ depending on the gravitino mass $m_{3 / 2}$.

According to the analysis [36, the upper bounds corresponding to a heavy, i.e unstable gravitino are given by $T_{R} \lesssim 10^{7}, 10^{9}$ and $10^{12} \mathrm{GeV}$ for $m_{3 / 2}=100 \mathrm{GeV}, 1 \mathrm{TeV}$ and $3 \mathrm{TeV}$, respectively ${ }^{3}$. Assuming that the right-handed neutrinos are produced thermally after inflation results in a constraint on $M_{1}$ [24], $M_{1}<10 T_{R}$. This potential problem of thermal leptogenesis can be overcome e.g. in anomaly or gaugino mediated supersymmetry, as has been realized in [38, 39], where the gravitino either decays before the initiation of nucleosynthesis, or becomes the stable LSP. Since the gauge couplings decrease above a critical temperature $T_{*}$, depending on the SUSY breaking scale, this mechanism leads to a relic gravitino density which is compatible with the WMAP results and which becomes independent of the reheating temperature for $T_{R}>T_{*}$. In any case a small reheating temperature and Majorana mass $M_{1}$ are desirable. We thus study in the following the values of the $R$ matrix in (48) for which $M_{1}$ acquires its minimum value.

\section{Constraints on the $R$ matrix}

As outlined in Section 2, the $R$ matrix (11) can be parametrized by six real parameters $x_{i}, y_{i}, i=1,2,3$. With the $x_{i}$ and $y_{i}$ given, $M_{1}$ is fixed by the leptogenesis condition (43). In order not to violate the gravitino bound on the reheating temperature in mSUGRA models, we require $M_{1}<10^{11} \mathrm{GeV}$. In addition, $\tilde{m}_{1}$ has been demanded to lie in the interval $\sqrt{\Delta m_{12}^{2}}<\tilde{m}_{1}<\sqrt{\Delta m_{23}^{2}}$, so that the fit (45) for the washout factor $\kappa_{f}$ is appropriate. Another important condition for obtaining meaningful results on physical observables is the reliability of perturbative theory in the Higgs sector. We therefore require that the

\footnotetext{
${ }^{3}$ More stringent bounds on the reheating temperature can be found in the recent analysis [37.
} 
largest Yukawa coupling eigenvalue $\left(Y_{\nu}\right)_{3}$ meets the condition $\left|\left(Y_{\nu}\right)_{3}\right|^{2} / 4 \pi \lesssim 0.3$. The largest Yukawa coupling $\left|\left(Y_{\nu}\right)_{3}\right|$ is very sensitive to the heaviest Majorana mass $M_{3}$ and the parameters $y_{i}$ in the $R$ matrix. This behavior can be easily understood from (10) and the parametrization (11) which imply a $\sqrt{M_{3}}$ dependence and an exponential dependence on $y_{i}$. Thus the perturbativity bound constrains $y_{i}<\mathcal{O}(1)$. In the subsequent numerical treatment an upper bound $y_{i}<1$ has been imposed and remaining points with values of $\left(Y_{\nu}\right)_{3}^{2} / 4 \pi>0.3$ have been rejected.

In the right-handed neutrino sector the following assumptions have been made. The $R$ matrix parameters $x_{1}, x_{2}$ and $x_{3}$ are varied in their full range $[0,2 \pi]$ and the imaginary parts $y_{i}$ are varied in the interval $\left[10^{-3}, 1\right]$. For larger values of $y_{i}$, the Higgs sector becomes strongly interacting. On the other hand, all three parameters $y_{i}$ have to be non-zero to make leptogenesis possible. Finally the hierarchical spectrum of the right-handed neutrinos has been generated by scattering $M_{3}$ logarithmically between $10 M_{1}$ and $10^{4} M_{1}$ and varying $M_{2}$ between $M_{1}$ and $0.1 M_{3}$. The condition $M_{2}>3 M_{1}$ required for (42) to hold is met for the predominant majority of scatter points.

The constraints on the $R$ matrix imposed by the requirement of a minimal Majorana mass $M_{1}$ for a given baryon asymmetry can be read off from (43). It is obvious that $M_{1}$ obtains its minimum values for $\Im m\left(R_{1 i}\right) \simeq \Re e\left(R_{1 i}\right)$, and thus a small $\Re e\left(R_{1 i}\right)$, since the imaginary parts are determined by the parameters $y_{i}$. While the contribution of $R_{11}$ in the sum in (48) is suppressed by the small value of $m_{1}$ in hierarchical neutrino spectra, $\Re e\left(R_{12}\right)$ and $\Re e\left(R_{13}\right)$ become small for $x_{2,3} \simeq n \pi$ with $n \in \mathbb{N}$ independently from the value of $x_{1}$, as is obvious from (11). Thus, the remaining angle $x_{1}$ cannot be constrained in this way. This is physically understandable, as $x_{1}$ is related to the mixing between the right-handed neutrinos $\nu_{R_{2}}$ and $\nu_{R_{3}}$, which plays no major role in the decays of $\nu_{R_{1}}$.

The allowed ranges of $x_{2}$ and $x_{3}$ are shown in Fig. 1 for $M_{1}=10^{10,11,12} \mathrm{GeV}$ (left to right). All the other $R$ matrix and light neutrino parameters are scattered in their ranges as outlined above and as given in Table 1, respectively. Each dot represents a viable data point which respects all the above selection rules. Fig. 1 also shows contours of constant $M_{1}=10^{10,11,12} \mathrm{GeV}$ as illustration for which these parameters are fixed at specific values. This behavior is also nicely illustrated in Fig. 2, which shows the decrease of the allowed range of $x_{2}$ with decreasing $M_{1}$. Above a value of $M_{1} \approx 1.6 \cdot 10^{11} \mathrm{GeV}, x_{2}$ can no longer be constrained and the full parameter range is allowed. The other $R$ matrix angle $x_{3}$ behaves analogously. 

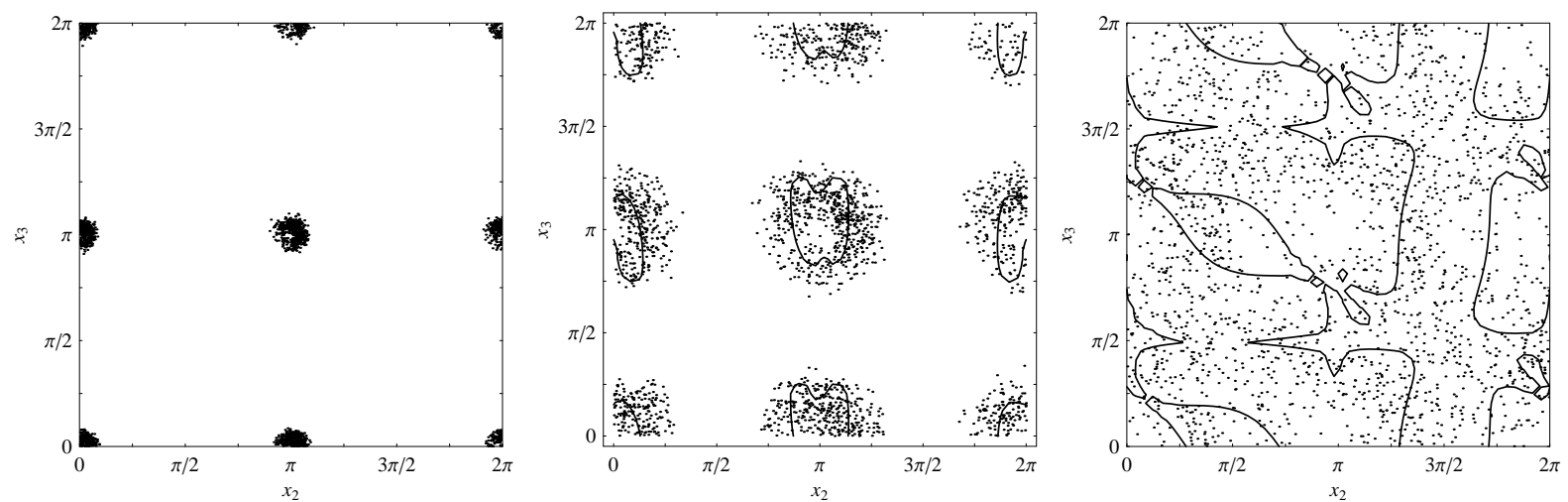

Figure 1: Regions in the plane $\left(x_{2}, x_{3}\right)$ consistent with the generation of the baryon asymmetry via leptogenesis and the gravitino bound $M_{1}=10^{10,11,12} \mathrm{GeV}$, from left to right, respectively. The procedure yielding the scatter plots is described in the text. Also shown are the contours of constant $M_{1}\left(x_{2}, x_{3}\right)=10^{10,11,12} \mathrm{GeV}$ with all other parameters fixed (best fit values of oscillation parameters, $m_{1}=0$, vanishing $\mathrm{CP}$ phases, $x_{1}=0$ and $y_{i}=0.2$, $i=1,2,3)$.

\section{Charged Lepton Flavor Violation}

In the previous sections the requirement of successful leptogenesis has been used to constrain the lightest Majorana mass $M_{1}$ and the parameters $y_{i}, x_{2,3}$ of the $R$ matrix. In the SUSY version of the seesaw model further observables are provided by the branching ratios of the radiative decays $l_{j} \rightarrow l_{i} \gamma$. As has been shown in [2] in the framework of degenerate Majorana neutrinos, these branching ratios are particularly sensitive to the Majorana mass scale. In the present framework of hierarchical Majorana neutrinos, a study of $\operatorname{Br}\left(l_{j} \rightarrow l_{i} \gamma\right)$ allows to obtain information about the heaviest Majorana mass $M_{3}$.

In the mass insertion approximation, the branching ratio $\operatorname{Br}\left(l_{j} \rightarrow l_{i} \gamma\right)$ is schematically given by [5, 6]

$$
B r\left(l_{j} \rightarrow l_{i} \gamma\right) \sim \frac{\alpha^{3} \tan ^{2} \beta}{\tilde{m}^{8}} \frac{m_{l_{j}}^{5}}{\Gamma_{j}}\left|\left(\delta m_{L}^{2}\right)_{j i}\right|^{2},
$$

where $\tilde{m}$ denotes the typical mass scale of the sleptons in the loop. For our numerical calculations, we use the complete one-loop results for $\operatorname{Br}\left(l_{j} \rightarrow l_{i} \gamma\right)$ [5], 16].

Some general conclusions can be drawn from the dependence on the five relevant, unknown seesaw parameters (see (15), (33) $) M_{3}, x_{1,2}$ and $y_{1,2}$,

$$
\left(\delta m_{L}^{2}\right)_{i j} \propto\left(Y_{\nu}^{\dagger} L Y_{\nu}\right)_{i j} \propto M_{3}\left|\cos \left(x_{2}+i y_{2}\right)\right|^{2} \log \frac{M_{X}}{M_{3}} f_{i j}\left(x_{1}+i y_{1}\right)
$$




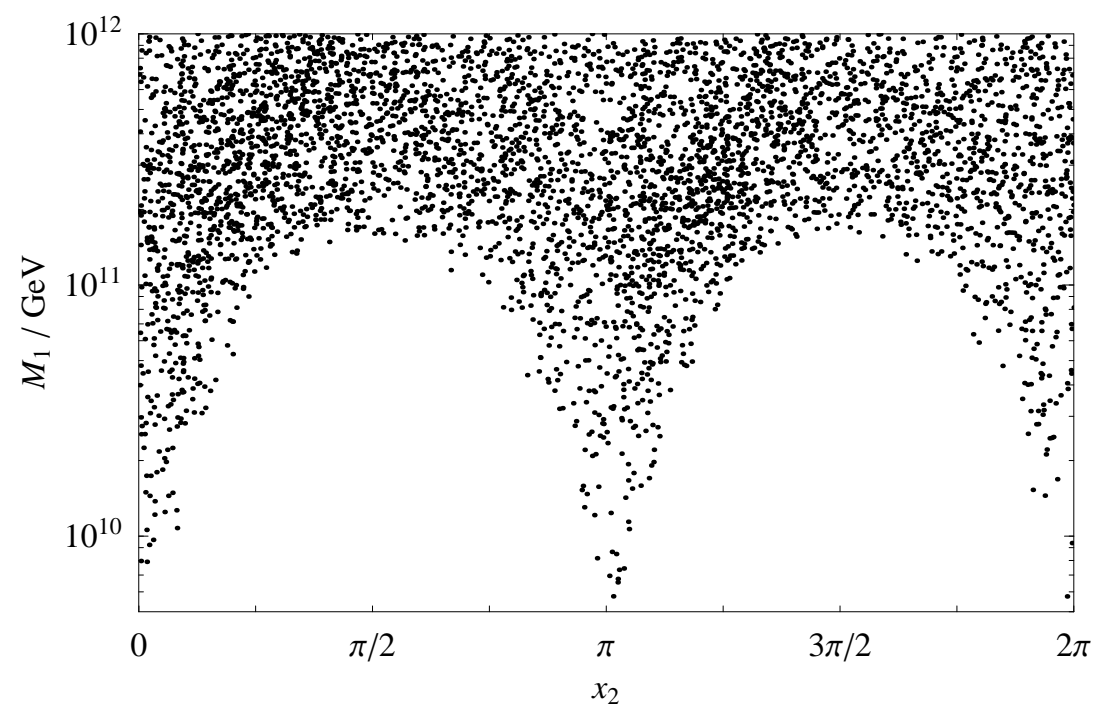

Figure 2: Region in the plane $\left(x_{2}, M_{1}\right)$ consistent with the generation of the baryon asymmetry via leptogenesis. The procedure yielding the scatter plot is described in the text.

where

$$
\begin{aligned}
& f_{12}=\left(\sqrt{\frac{m_{2}}{m_{3}}} \hat{s}_{1} V_{12}^{*}+\hat{c}_{1} V_{13}^{*}\right)^{*}\left(\sqrt{\frac{m_{2}}{m_{3}}} \hat{s}_{1} V_{22}^{*}+\hat{c}_{1} V_{23}^{*}\right), \\
& f_{23}=\left(\sqrt{\frac{m_{2}}{m_{3}}} \hat{s}_{1} V_{22}^{*}+\hat{c}_{1} V_{23}^{*}\right)^{*}\left(\sqrt{\frac{m_{2}}{m_{3}}} \hat{s}_{1} V_{32}^{*}+\hat{c}_{1} V_{33}^{*}\right) .
\end{aligned}
$$

These expressions can be contrasted with the present experimental limits 40, 41,

$$
\begin{aligned}
& \operatorname{Br}(\mu \rightarrow e \gamma)<1.2 \cdot 10^{-11} \\
& \operatorname{Br}(\tau \rightarrow \mu \gamma)<6.8 \cdot 10^{-8}
\end{aligned}
$$

as well as the expected sensitivity of the MEG experiment at PSI [42] and data from future B-factories [41],

$$
\begin{aligned}
& B r(\mu \rightarrow e \gamma) \simeq 10^{-13} \\
& B r(\tau \rightarrow \mu \gamma) \simeq 10^{-8} .
\end{aligned}
$$

From (49 50) it is immediately obvious that the branching ratios increase with the square of the heaviest Majorana mass $M_{3}$. This behavior is illustrated in Fig. 3 for $M_{1}=10^{10,11,12} \mathrm{GeV}$ (left to right) and provides a possibility to determine $M_{3}$ from the combination of parameters $M_{3}\left|\cos \theta_{2}\right|^{2} \simeq M_{3}$. The present bound on $\operatorname{Br}(\mu \rightarrow e \gamma)$ constrains $M_{3}$ already to be smaller than some $10^{13} \mathrm{GeV}$. The sensitivity of the future MEG experiment at PSI would allow to 
bound $M_{3}<\mathcal{O}\left(10^{12}\right) \mathrm{GeV}$ or determine the value of $M_{3}$ with an accuracy of a factor of 10 . Due to the hierarchy $M_{3} / M_{1} \geq 10$ used in our scattering, the minimum value for $M_{3}$ rises with $M_{1}$, pushing more and more points above the present limit on the branching ratio. The widening of the scatter range for $M_{1}<10^{12} \mathrm{GeV}$ (third plot) is largely due to the fact that $x_{2}$ is no longer constrained around $n \pi$, as can be seen in the third plot of Fig. 1 . Hence, $\operatorname{Br}(\mu \rightarrow e \gamma) \propto\left|\cos \theta_{2}\right|^{2}$ can be strongly suppressed.

The dependence $\operatorname{Br}\left(l_{j} \rightarrow l_{i} \gamma\right)$ on the initial and final flavor is encoded in the functions $f_{i j}$, which show a sensitive variation with the value of $x_{1}$. In particular, $f_{12}$ and $f_{23}$ given in (5152) have minima at small $y_{1}$ and different values of $x_{1}$, which imply a strong variation of the ratio $\operatorname{Br}(\mu \rightarrow e \gamma) / \operatorname{Br}(\tau \rightarrow \mu \gamma)$ with $x_{1}$. This ratio, which is rather independent of the mSUGRA scenario used (33449), is plotted in Fig. 4. The dependence on $M_{3} \cos ^{2} \theta_{2}$ drops out and a measurement of both $\operatorname{Br}(\mu \rightarrow e \gamma)$ and $\operatorname{Br}(\tau \rightarrow \mu \gamma)$ would allow to determine $x_{1}$ with an accuracy of about $\mathcal{O}(1)$. It should be stressed, though, that present (5354) and planned (5556) experiments have a very restricted sensitivity, $\operatorname{Br}(\mu \rightarrow e \gamma) / \operatorname{Br}(\tau \rightarrow \mu \gamma)<$ $\mathcal{O}\left(10^{-3}\right)$. If future B-factories would observe $\tau \rightarrow \mu \gamma$, the angle $x_{1}$ could also be restricted to values $x_{1} \approx n \pi$.

\section{Conclusions}

We have studied the minimal supersymmetric seesaw model by imposing constraints from light neutrino data, charged lepton flavor violation, successful leptogenesis and perturbativity in the neutrino sector. The parameters describing the light neutrino sector have been fixed to their experimental best fit values with an uncertainty anticipated from planned future experiments, and a very light neutrino spectrum, $m_{1} \lesssim m_{2} \lesssim m_{3}$ with $m_{1}<0.03 \mathrm{eV}$ has been assumed.

The lightest right-handed neutrino mass $M_{1}$ is fixed by the CP asymmetry $\epsilon_{1}$ imposed to generate the observed value $\eta_{B}=(6.3 \pm 0.3) \cdot 10^{-10}$ for the baryon asymmetry and the requirement of a small reheating temperature with $M_{1}<10 T_{R}<\mathcal{O}\left(10^{11}\right) \mathrm{GeV}$. The largest Majorana mass $M_{3}$ is constrained from above by the LFV process $\mu \rightarrow e \gamma$. Thus the right-handed neutrino spectrum can be summarized as

$$
\mathcal{O}\left(10^{11}\right) \mathrm{GeV} \sim M_{1}<M_{2}<M_{3} \lesssim \mathcal{O}\left(10^{13}\right) \mathrm{GeV}
$$

in the given SUSY scenario SPS1a. Alternative SUSY scenarios with sparticle masses in the range $100 \mathrm{GeV}-1 \mathrm{TeV}$ and low $\tan \beta$ are expected to yield similar results. 

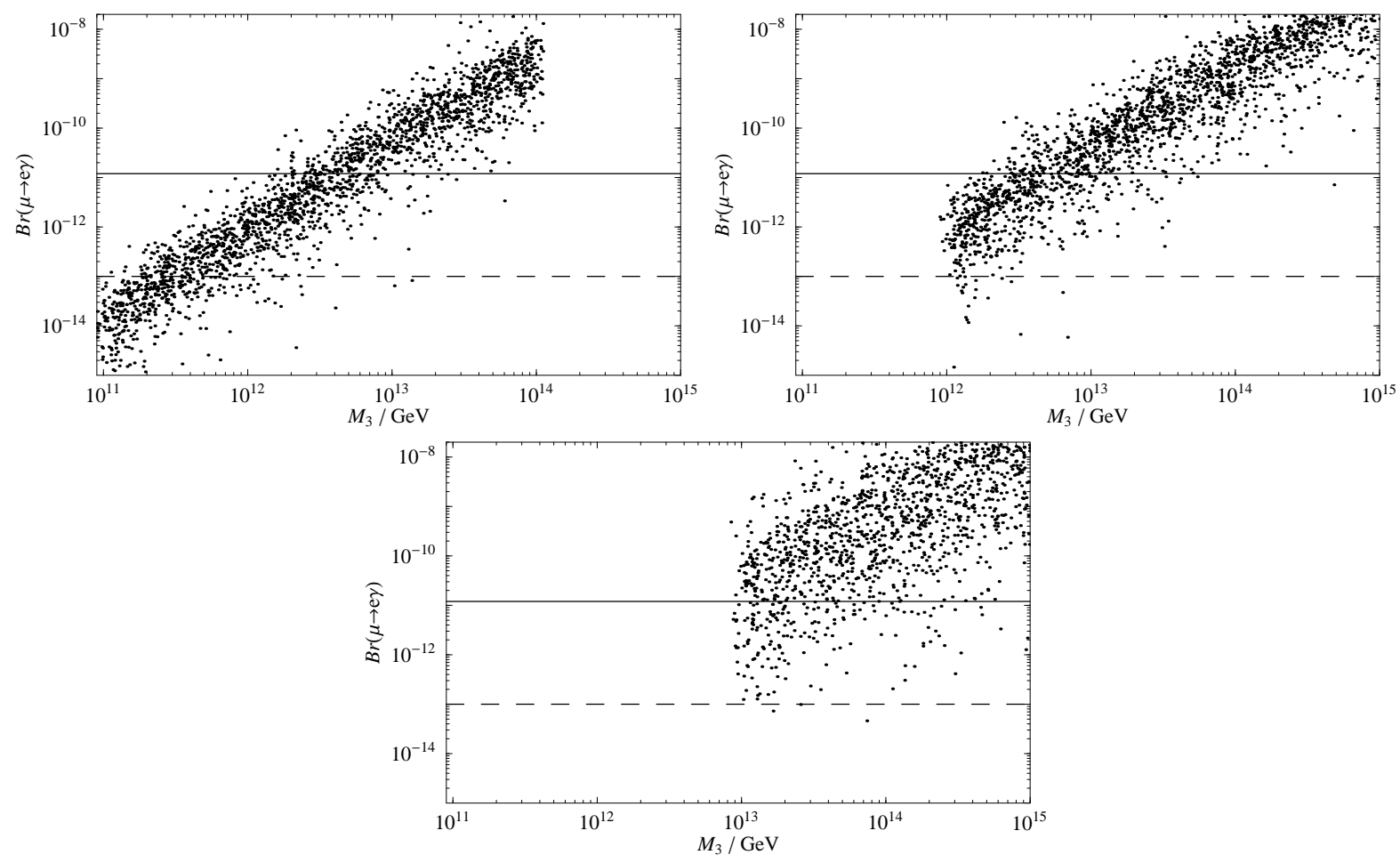

Figure 3: Branching ratio of $\mu \rightarrow e \gamma$ as a function of the heaviest Majorana mass $M_{3}$ in SUSY scenario SPS1a, for $M_{1}=10^{10,11,12} \mathrm{GeV}$, respectively. The solid (dashed) line indicates the present (expected future) experimental sensitivity. For a description of the scatter procedure see text.

In addition the successful scenario possesses an orthogonal $R$ matrix encoding the mixing of the right-handed neutrinos, which is parametrized by the angles $\theta_{i}=x_{i}+i y_{i}$ with small but non-vanishing imaginary parts, being constrained as

$$
\begin{aligned}
x_{2,3} & \simeq n \pi, \quad n \in \mathbb{N}, \\
0 & <y_{i} \lesssim \mathcal{O}(1) .
\end{aligned}
$$

These bounds on $y_{i}, x_{2,3}$ result from the requirement of perturbative Yukawa couplings and the condition of successful leptogenesis under the constraint of a small $M_{1}$. The remaining parameter $x_{1}$ can be determined from the ratio $\operatorname{Br}(\mu \rightarrow e \gamma) / \operatorname{Br}(\tau \rightarrow \mu \gamma)$.

\section{Note Added}

When this paper has been accomplished the preprint 43] appeared, which comes to similar conclusions. One important difference between the two studies is that the authors of 43 . 


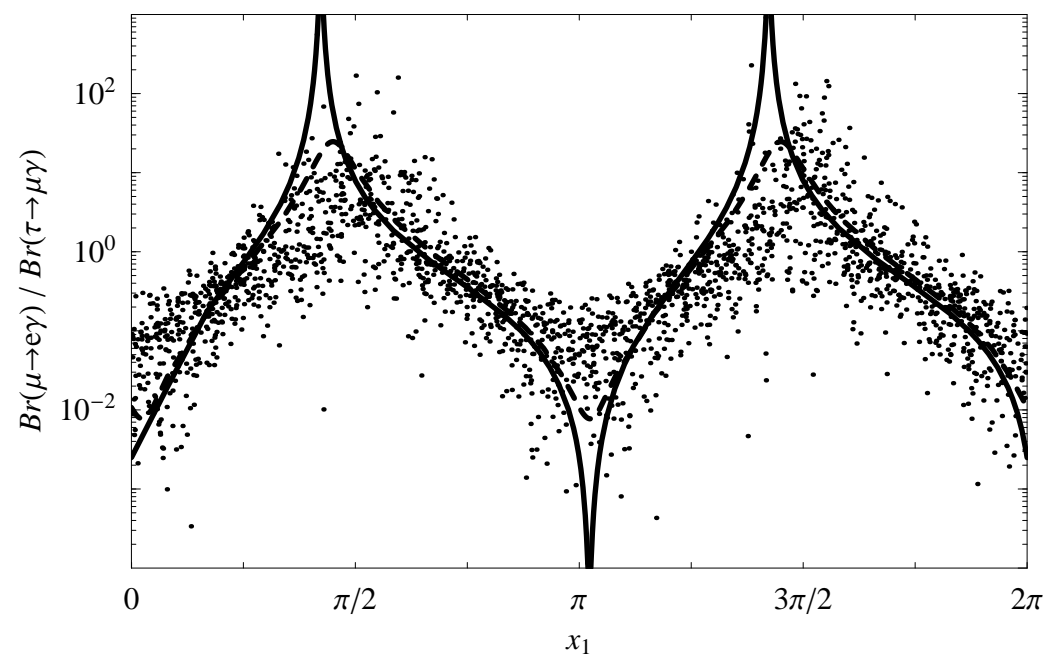

Figure 4: Ratio $\operatorname{Br}(\mu \rightarrow e \gamma) / \operatorname{Br}(\tau \rightarrow \mu \gamma)$ as a function of $x_{1}$ in SUSY scenario SPS1a and for $M_{1}=10^{11} \mathrm{GeV}$. For a description of the scatter procedure see text. Superimposed are two curves for which all parameters other than $x_{1}$ are fixed (best fit oscillation parameters, $m_{1}=0 \mathrm{eV}$, no CP phases and $y_{2,3}=0$ ). The solid (dashed) curve corresponds to $y_{1}=0.01$ $\left(y_{1}=0.1\right)$.

made further theoretical assumptions leading to more definite but less general results.

\section{Acknowledgements}

We thank W. Buchmüller and M. Plümacher for many useful comments and discussions. This work was supported by the Bundesministerium für Bildung und Forschung (BMBF, Bonn, Germany) under the contract number 05HT1WWA2 and by US DOE under the grant DE-FG03-91ER40833.

\section{References}

[1] H. Fritzsch, M. Gell-Mann and P. Minkowski, Phys. Lett. B 59, 256 (1975); H. Fritzsch and P. Minkowski, Phys. Lett. B 62, 72 (1976); P. Minkowski, Phys. Lett. B 67, 421 (1977); M. Gell-Mann, P. Ramond and R. Slansky, Proceedings of the Supergravity Stony Brook Workshop, New York 1979, eds. P. Van Nieuwenhuizen and D. Freedman; T. Yanagida, Proceedings of the Workshop on Unified Theories and Baryon Number in the Universe, Tsukuba, Japan 1979, eds. A. Sawada and A. Sugamoto; S. L. Glashow, Proceedings of the 1979 Cargèse Summer Institute on Quarks and Leptons eds. M. Lévy, 
J.-L. Basdevant, D. Speiser, J. Weyers, R. Gastmans, and M. Jacob, Plenum Press, New York, 1980, pp. 687-713; R. N. Mohapatra and G. Senjanovic, Phys. Rev. Lett. 44, 912 (1980), erratum ibid Phys. Rev. D 23, 165 (1981); J. Schechter and J. W. F. Valle, Phys. Rev. D 22, 2227 (1980); J. Schechter and J. W. F. Valle, Phys. Rev. D 25, 774 (1982).

[2] F. Deppisch, H. Päs, A. Redelbach, R. Rückl and Y. Shimizu, Eur. Phys. J. C 28, 365 (2003) [arXiv hep-ph/0206122.

[3] F. Deppisch, H. Päs, A. Redelbach, R. Rückl and Y. Shimizu, Phys. Rev. D 69, 054014 (2004) [arXiv hep-ph/0310053.

[4] F. Borzumati and A. Masiero, Phys. Rev. Lett. 57, 961 (1986); J. Hisano, D. Nomura and T. Yanagida, Phys. Lett. B 437, 351 (1998) [arXiv hep-ph/9711348; J. Hisano and D. Nomura, Phys. Rev. D 59, 116005 (1999) [arXiv:hep-ph/9810479]; K. S. Babu, B. Dutta and R. N. Mohapatra, Phys. Lett. B 458, 93 (1999) [arXiv hep-ph/9904366; K. S. Babu, B. Dutta and R. N. Mohapatra, Phys. Rev. D 67, 076006 (2003) [arXiv:hep-ph/0211068. B. Dutta and R. N. Mohapatra, Phys. Rev. D 68, 056006 (2003) [arXiv:hep-ph/0305059. W. Buchmuller, D. Delepine and F. Vissani, Phys. Lett. B 459, 171 (1999) [arXiv:hep-ph/9904219]; J. R. Ellis, M. E. Gomez, G. K. Leontaris, S. Lola and D. V. Nanopoulos, Eur. Phys. J. C 14, 319 (2000) [arXiv hep-ph/9911459; J. Hisano and K. Tobe, Phys. Lett. B 510, 197 (2001) [arXiv hep-ph/0102315; D. F. Carvalho, J. R. Ellis, M. E. Gomez, S. Lola and J. C. Romao, Phys. Lett. B 618, 162 (2005) [arXiv:hep-ph/0206148]; S. Lavignac, I. Masina and C. A. Savoy, Phys. Lett. B 520, 269 (2001) [arXiv hep-ph/0106245]; Y. Kuno and Y. Okada, Rev. Mod. Phys. 73, 151 (2001) [arXiv hep-ph/9909265]; J. R. Ellis, J. Hisano, M. Raidal and Y. Shimizu, Phys. Rev. D 66, 115013 (2002) [arXiv:hep-ph/0206110; A. Dedes, J. R. Ellis and M. Raidal, Phys. Lett. B 549, 159 (2002) [arXiv hep-ph/0209207; J. Hisano, arXiv:hep-ph/0209005 S. Pascoli, S. T. Petcov and C. E. Yaguna, Phys. Lett. B 564, 241 (2003) [arXiv:hep-ph/0301095]; T. Fukuyama, T. Kikuchi and N. Okada, Phys. Rev. D 68, 033012 (2003) [arXiv:hep-ph/0304190; J. I. Illana and M. Masip, Eur. Phys. J. C 35, 365 (2004) [arXiv:hep-ph/0307393; A. Masiero, S. K. Vempati and O. Vives, New J. Phys. 6, 202 (2004) [arXiv hep-ph/0407325; E. Arganda and M. J. Herrero, arXiv hep-ph/0510405; E. Arganda, A. M. Curiel, M. J. Herrero and D. Temes, Phys. Rev. D 71, 035011 (2005) [arXiv hep-ph/0407302; 
P. H. Chankowski, J. R. Ellis, S. Pokorski, M. Raidal and K. Turzynski, Nucl. Phys. B 690, 279 (2004) [arXiv hep-ph/0403180.

[5] J. Hisano, T. Moroi, K. Tobe and M. Yamaguchi, Phys. Rev. D 53, 2442 (1996) [arXiv:hep-ph/9510309

[6] J. A. Casas and A. Ibarra, Nucl. Phys. B 618171 (2001) [arXiv hep-ph/0103065.

[7] D. N. Spergel et al., Astrophys. J. Suppl. 148, 175 (2003) [arXiv astro-ph/0302209.

[8] M. Tegmark et al. [SDSS Collaboration], Phys. Rev. D 69, 103501 (2004) [arXiv:astro-ph/0310723].

[9] M. Fukugita and T. Yanagida, Phys. Lett. B 174, 45 (1986).

[10] S. Davidson and A. Ibarra, Phys. Lett. B 535, 25 (2002) [arXiv hep-ph/0202239.

[11] J. R. Ellis and M. Raidal, Nucl. Phys. B 643, 229 (2002) [arXiv:hep-ph/0206174.

[12] S. Pascoli, S. T. Petcov and W. Rodejohann, Phys. Rev. D 68, 093007 (2003) [arXiv:hep-ph/0302054.

[13] A. Pilaftsis, Int. J. Mod. Phys. A 14, 1811 (1999) [arXiv hep-ph/9812256]; Phys. Rev. D 56, 5431 (1997) [arXiv:hep-ph/9707235]; Nucl. Phys. B 504, 61 (1997) [arXiv:hep-ph/9702393.

[14] T. Hambye, Y. Lin, A. Notari, M. Papucci and A. Strumia, Nucl. Phys. B 695, 169 (2004) [arXiv hep-ph/0312203.

[15] S. Antusch, J. Kersten, M. Lindner and M. Ratz, Phys. Lett. B 538, 87 (2002) [arXiv:hep-ph/0203233.

[16] S. P. Martin and M. T. Vaughn, Phys. Rev. D 50, 2282 (1994) [arXiv hep-ph/9311340].

[17] W. de Boer, Prog. Part. Nucl. Phys. 33, 201 (1994) [arXiv hep-ph/9402266].

[18] B. C. Allanach et al., Eur. Phys. J. C 25 (2002) 113 [arXiv hep-ph/0202233]; H.U. Martyn, arXiv:hep-ph/040612 [LC-PHSM-2003-071].

[19] J. A. Aguilar-Saavedra et al., arXiv:hep-ph/0511344.

[20] M. Maltoni, T. Schwetz, M. A. Tortola and J. W. F. Valle, Phys. Rev. D 68, 113010 (2003) [arXiv hep-ph/0309130. 
[21] A. Strumia and F. Vissani, Nucl. Phys. B 726, 294 (2005) [arXiv hep-ph/0503246].

[22] K. Matsuda, T. Fukuyama and H. Nishiura, Mod. Phys. Lett. A 18, 1803 (2003) [arXiv,hep-ph/0302254; F. Deppisch, H. Päs and J. Suhonen, Phys. Rev. D 72, 033012 (2005) [arXiv/hep-ph/0409306]; S. Pascoli, S. T. Petcov and T. Schwetz, arXiv hep-ph/0505226; and references therein.

[23] H. Päs and T. J. Weiler, Phys. Rev. D 63, 113015 (2001) [arXiv hep-ph/0101091].

[24] P. Di Bari, arXiv hep-ph/0406115, P. Di Bari, Nucl. Phys. B 727, 318 (2005) [arXiv hep-ph/0502082.

[25] W. Buchmüller, P. Di Bari and M. Plümacher, Nucl. Phys. B 643, 367 (2002) [arXiv:hep-ph/0205349]; W. Buchmuller, P. Di Bari and M. Plumacher, Nucl. Phys. B 665, 445 (2003) [arXiv hep-ph/0302092.

[26] W. Buchmuller, P. Di Bari and M. Plumacher, arXiv hep-ph/0401240.

[27] M. Plümacher, arXiv hep-ph/9807557 and references therein.

[28] L. Covi, E. Roulet and F. Vissani, Phys. Lett. B 384, 169 (1996) [arXiv:hep-ph/9605319].

[29] W. Buchmüller and M. Plümacher, Int. J. Mod. Phys. A 15, 5047 (2000) [arXiv hep-ph/0007176.

[30] K. Hamaguchi, H. Murayama and T. Yanagida, Phys. Rev. D 65, 043512 (2002) [arXiv:hep-ph/0109030; R. Barbieri, P. Creminelli, A. Strumia and N. Tetradis, Nucl. Phys. B 575, 61 (2000) [arXiv hep-ph/9911315.

[31] M. Hirsch and S. F. King, Phys. Rev. D 64, 113005 (2001) [arXiv hep-ph/0107014.

[32] H. B. Nielsen and Y. Takanishi, Phys. Lett. B 507, 241 (2001) [arXiv hep-ph/0101307].

[33] P. Di Bari, AIP Conf. Proc. 655, 208 (2003) [arXiv hep-ph/0211175].

[34] M. Y. Khlopov and A. D. Linde, Phys. Lett. B 138, 265 (1984); J. R. Ellis, J. E. Kim and D. V. Nanopoulos, Phys. Lett. B 145, 181 (1984); I. V. Falomkin, G. B. Pontecorvo, M. G. Sapozhnikov, M. Y. Khlopov, F. Balestra and G. Piragino, Nuovo Cim. A 79, 193 (1984) [Yad. Fiz. 39, 990 (1984)]; M. Y. Khlopov, Y. L. Levitan, E. V. Sedelnikov and I. M. Sobol, IC-93-191; M. Y. Khlopov and A. Barrau, arXiv astro-ph/0406621. 
[35] K. Hamaguchi, arXiv hep-ph/0212305, and references therein.

[36] M. Kawasaki, K. Kohri and T. Moroi, Phys. Rev. D 63, 103502 (2001) [arXiv:hep-ph/0012279].

[37] K. Kohri, T. Moroi and A. Yotsuyanagi, arXiv/hep-ph/0507245.

[38] T. Gherghetta, G. F. Giudice and J. D. Wells, Nucl. Phys. B 559, 27 (1999) [arXiv hep-ph/9904378].

[39] W. Buchmüller, K. Hamaguchi and M. Ratz, Phys. Lett. B 574, 156 (2003) [arXiv:hep-ph/0307181].

[40] M. Hebert [MECO Collaboration], Nucl. Phys. A 721, 461 (2003);

[41] B. Aubert et al. [BABAR Collaboration], Phys. Rev. Lett. 95, 041802 (2005) [arXiv:hep-ex/0502032.

[42] MEG experiment home page: http://meg.psi.ch

[43] S. T. Petcov, W. Rodejohann, T. Shindou and Y. Takanishi, arXiv hep-ph/0510404. 